\title{
Recherches-actions associatives : Le praticien réflexif ou la recherche sans « chercheur »
}

\section{Dominique Cottereau}

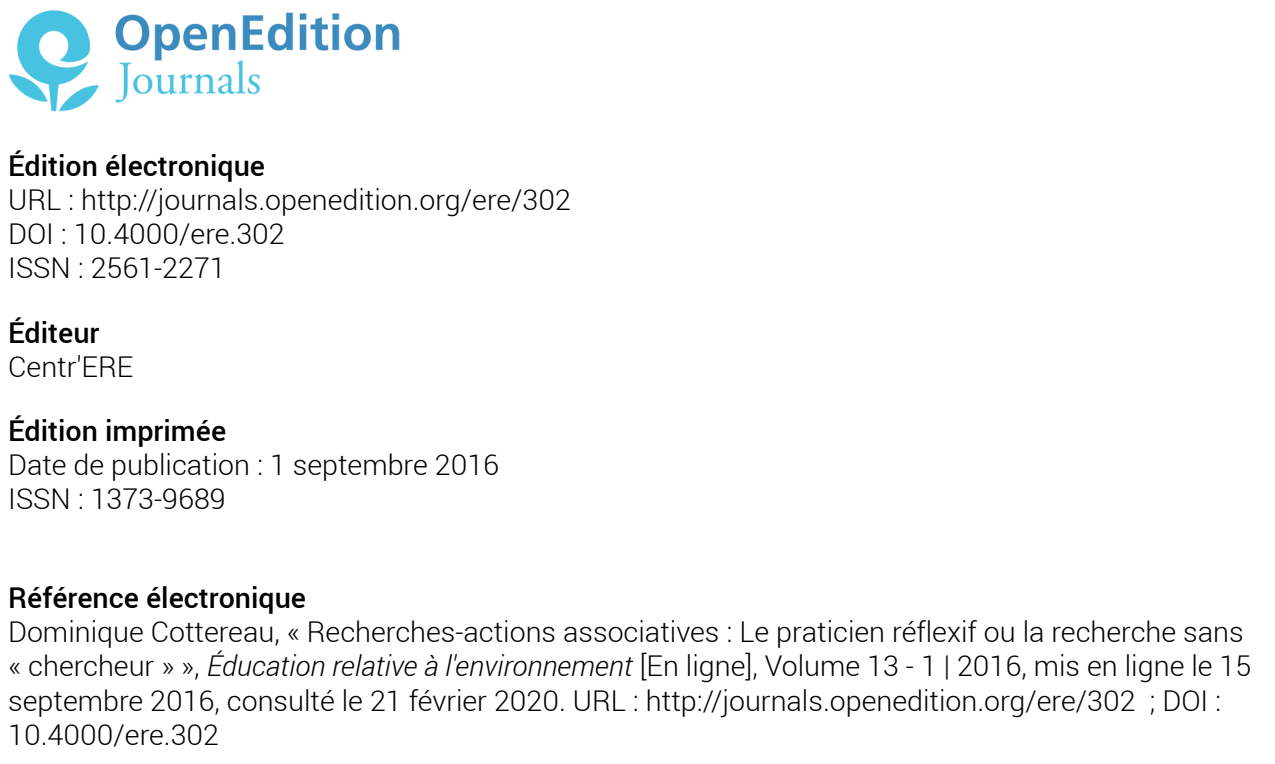

Ce document a été généré automatiquement le 21 février 2020. 


\title{
Recherches-actions associatives : Le praticien réflexif ou la recherche sans « chercheur »
}

\author{
Dominique Cottereau
}

1 L'hypothèse d'un «tiers secteur scientifique » (Latour, 2004) émerge à la fois dans des laboratoires de recherche, dans des communautés citoyennes ou des programmes politiques de financement de recherches collaboratives. Les programmes PICRI en Ile de France (Partenariats Institutions Citoyens pour la Recherche et l'Innovation), ASOSC en Bretagne (Appropriation Sociale des Sciences) ${ }^{1}$, tous deux inspirés des ARUC québécoises (Alliances pour la Recherche Universités et Communautés) témoignent de ce mouvement social en train d'inventer de nouvelles formes de recherches où les positions respectives des scientifiques et des acteurs ne sont plus si tranchées que dans les recherches centrées sur le chercheur et ses objets (Goffin, 1998-1999). Il y a là l'hypothèse que l'acteur apporte tout autant que le chercheur dans une recherche soulevée par une question de société. L'acteur connait son terrain, le nomme de ses propres savoirs (qu'on les appelle savoirs profanes, vernaculaires, quotidiens, ordinaires, autochtones, expérientiels...). Immergé dans sa pratique sociale, il porte un non-dit et un insu des gestes plus riches que ce que le chercheur peut en présumer. Le chercheur, de son côté, apporte ses savoirs scientifiques, sa méthodologie, sa mise en perspective, la possibilité de la reconnaissance des résultats par ses pairs. Mais qu'en est-il de ce tiers secteur lorsqu'il n'y a pas de chercheur habilité par un laboratoire de recherche? Quelle légitimité scientifique acquièrent ces recherches menées plutôt par des praticiens réflexifs? Ne profitent-elles pas surtout aux acteurs sans entrer dans la boucle des communications académiques par difficulté d'être reconnues comme recherches à part entière ?

2 J'explorerai ce thème de la recherche associative à partir d'une expérience menée par la Plateforme bretonne de l'Éducation à l'environnement et au développement durable (EEDD) qui a tenté d'insuffler des recherches-actions coopératives sur son territoire. La plateforme est une instance informelle de pilotage d'un plan d'action pour le 
développement de l'éducation à l'environnement et au développement durable. Coanimée par le Réseau d'éducation à l'environnement en Bretagne (REEB) et la Direction Régionale de l'Environnement, de l'Aménagement et du Logement (DREAL), elle réunit des services et agences de l'État (Direction Régionale de la Jeunesse, des Sports et de la Cohésion Sociale, Rectorat, Direction Régionale de l'Alimentation, de l'Agriculture et de la Forêt, Agence de Développement de l'Environnement et de la Maîtrise de l'Énergie, Agence de l'eau Loire Bretagne), la Région Bretagne et les Conseils généraux d'Ille-etVilaine, du Finistère, des Côtes-d'Armor et du Morbihan. En 2011-2012, quatre recherches-actions ont été menées, financées par la DREAL, pilotées par la plateforme, accompagnées par moi-même. Je n'appartenais à aucun laboratoire de recherche et étais habilitée à cet accompagnement par la DREAL en tant que salariée d'une association soutenue par cette institution. Nous étions dans ce cas de figure de recherches totalement déconnectées des universités et laboratoires officiels.

3 J'aurais pu faire de cette expérience l'objet d'une recherche personnelle en posant un cadre théorique et une méthodologie avant la mise en œuvre du dispositif. Ce ne fut pas le cas notamment parce que je n'étais inscrite dans aucun projet de recherche lié aux démarches de recherches-actions associatives. Mon seul but était alors d'initier la recherche-action chez les acteurs de terrain, tout en les formant aux méthodologies de la recherche. Ce n'est qu'après coup, sur invitation de l'Institut de formation et de recherche en éducation à l'environnement (Ifrée) dans le cadre du symposium qu'il organisait en décembre 2013, que j'ai décidé de porter une analyse réflexive sur le dispositif et d'en tirer des enseignements à un niveau microsocial. Je me suis alors constitué un cadre théorique à partir de travaux en sociologie de la participation (Latour, Callon, Nez, Souchard), de «la traduction» (Callon, Audou et Gillet), sur la recherche-action (Sauvé, Barbier, Galvani) et sur le «praticien réflexif» (Schön). J'ai suivi une double méthodologie, celle de l'explicitation de l'expérience nourrie de l'éclairage conceptuel, et je suis allée questionner les porteurs de projets de rechercheaction pour en connaitre les conséquences et vérifier si la production de savoirs avait été utile à la science et/ou à l'action.

4 Je présenterai d'abord le contexte d'émergence de cette initiative. Je clarifierai mon rôle de chercheure au sein de la plateforme et des expérimentations. Je décrirai les recherches-actions. Pour finir, je synthétiserai les freins d'une recherche-action associative qui ne s'inscrit pas dans un processus de partenariat avec un laboratoire de recherche statutaire.

\section{Contexte : le Plan régional d'action en Bretagne}

5 Le Collectif régional de l'éducation à l'environnement est né dans le contexte social de mise en réseau des acteurs. Après le sommet international francophone "Planet'ERE " de 2001 organisé en France et la parution d'un Plan national d'action, les acteurs bretons souhaitèrent se doter d'un plan régional adapté aux caractéristiques et aux enjeux de leur territoire. En 2003, une méthodologie participative de co-élaboration de ce plan fut mise en place, animée par le REEB. Pendant deux ans se succédèrent des séances de travail tantôt en comité de pilotage, tantôt en groupe technique, tantôt en plénière de plus de cent participants, avec circulations intermédiaires de comptes rendus et de propositions dans tout le réseau. Il en est résulté un document de vingt-etune pages, conçu de près ou de loin par plus de deux cents personnes, inauguré le 21 
septembre 2005, et signé ce même jour par dix institutions. L'un des objectifs du Plan régional d'action ( . 15) portait sur le développement de la connaissance et de la recherche :

Toute pratique éducative doit être consciente d'elle-même, réfléchie, soucieuse de s'insérer dans les évolutions sociétales. La prise de recul sur son action et sur ses interférences avec le monde alentour est indispensable. Observer, enquêter, diagnostiquer, analyser, évaluer, expérimenter... sont autant d'actions nécessaires à une éducation à l'environnement maîtrisée, adaptée, cohérente et performante.

Or aucune action ne semblait mise en place pour y répondre. La plateforme, chargée de soutenir et mettre en œuvre le plan, décida de lui accorder du temps de réflexion.

7 J'animais ce travail au nom du REEB, en tant qu'administratrice bénévole de l'association. Les résultats du colloque "Écocitoyenneté, lieu d'échanges entre chercheurs et praticiens", organisé à Marseille les 18 et 19 novembre 2004 nous servirent d'embrayeur de désir. Pendant trois années, nous avons tenté de définir nos orientations autour de quelques questions: qu'est-ce qu'on entend par rechercheaction? Quels acteurs du territoire breton mènent des recherches susceptibles d'intéresser l'EEDD? Quels pourraient être les objets de connaissance? Comment rassembler praticiens et chercheurs?

Nous nous entendons sur une définition de la recherche-action empruntée à la fois à Lucie Sauvé (1998-1999) et René Barbier (1996). Il s'agit bien d'une recherche avec ce qu'elle comporte habituellement de formulation d'une question problématisée, d'un état de l'art sur la question, d'un référencement théorique et bibliographique, la délimitation d'un terrain d'étude, la formulation d'une méthodologie d'investigation, la mise en ordre et l'interprétation des résultats, et une communication de ses résultats. Mais cette forme de recherche doit être fondée sur une réalité à transformer par l'action : la problématique est justifiée par une question émergeant de la réalité ; elle est interdisciplinaire, participative (et non descendante), avec prise de décision sur les changements à opérer. "La recherche-action n'est pas un simple avatar méthodologique de la sociologie classique. Elle exprime au contraire une véritable transformation de la manière de concevoir et de faire de la recherche en sciences humaines », comme le revendique René Barbier (1996, p. 7).

\section{L'accompagnateur chercheur engagé}

Cette définition de la recherche-action fut moins une construction collective qu'une proposition de ma part immédiatement adoptée par la plateforme. Les représentants des institutions reconnaissaient n'avoir pas de temps à consacrer à la question entre les réunions et laissaient le REEB piloter la réflexion, les recherches d'informations, l'élaboration des propositions. Certains se disaient également non légitimes, la recherche ne faisant partie ni de leurs missions ni des centres d'intérêts des élus qu'ils représentaient. Ils se prétendaient par ailleurs démunis en compétences.

10 Je dois ici faire un zoom d'explicitation sur ma place et mon rôle dans ce processus. Depuis 1994, je suis une adhérente activement engagée dans les réseaux associatifs de l'éducation à l'environnement. Et depuis l'obtention d'un doctorat en sciences de l'éducation, je tente d'insuffler une dynamique de recherche chez les praticiens. J'ai été moi-même praticienne doctorante, menant une thèse en formation continue par désir d'éclairer ma pratique d'éducatrice. Ce passage par la recherche bouleversa ma vision 
de l'éducation à l'environnement. Je découvrais cette posture fertile du praticien réflexif décrit par Schön (1994). Je réfléchissais mon acte pédagogique sur le miroir de la conceptualisation faisant de chaque théorie, de chaque concept exploré un outil de saisie signifiant de ma quotidienneté professionnelle que je transformais à la lumière de ces découvertes. Mon action prit un sens nouveau, personnellement plus congruent avec mon être-au-monde, et professionnellement plus cohérent avec nos finalités éducatives. L'enthousiasme de cette transformation me poussa à la partager avec la sphère associative de l'éducation à l'environnement (Cottereau, 2001), et notamment à impulser des recherches issues du terrain sur des problématiques d'acteurs en prise avec des questions de société, d'environnement, d'éducation/formation, des recherches qui seraient fondées «sur un appel issu de la pratique afin de produire de nouvelles connaissances » selon la définition de la recherche partenariale des sociologues Bussières et Fontant (2011, p. 5).

11 Au-delà même de ma subjectivité personnelle, je pensais qu'il était nécessaire d'entourer l'éducation à l'environnement d'une éthique et d'une vision éclairée sur ce qu'elle produit et comment elle le produit. La pression sociale et politique de plus en plus forte ainsi que le sentiment d'urgence sur les problèmes d'environnement ne doivent pas oblitérer les questions du sens. Les sociologues Chantal Aspe et Marie Jacqué ont analysé combien l'éducation à l'environnement, comme le projet écologique, sont passés, au fil de leur histoire, de la posture critique à celle de l'adaptation :

Présentée comme concourant à un projet de transformation de la société, la morale écologique s'inscrit pourtant dans un processus social d'intégration normative et idéologique de la question environnementale, alors qu'elle est, la plupart du temps, pensée et vécue par les individus comme critique du mode de développement dominant. (Aspe et Jacqué, 2012, p. 214)

12 La recherche permet de gagner en lucidité sur les jeux politiques et sociaux dans lesquels se trouve plongée l'éducation à l'environnement. Elle éclaire les multiples interférences entre acteurs, société, pédagogies, publics, nature. Elle aide au choix des orientations et à la prise de décision.

13 Je restais toutefois salariée d'une association dans laquelle je tentais d'animer toutes nos expérimentations pédagogiques ainsi que les diverses études que je conduisais en tant que consultante avec des méthodes rigoureuses et l'appel aux savoirs scientifiques (par exemple, Cottereau et Le Goff, 2012). J'étais ponctuellement chargée de cours à l'université François-Rabelais de Tours et celle de Rennes 2, je participais à un groupe informel de recherche sur l'écoformation, le GREF, coordonné par deux chercheurs en sciences de l'éducation, Gaston Pineau et René Barbier. Nous produisions régulièrement des ouvrages. Je rédigeais des articles dans des revues professionnelles et des revues à comité de lecture scientifique. J'acquérais une reconnaissance auprès de mes pairs, mais doutais de ma légitimité de chercheur au sein du monde scientifique. J'étais installée dans cette tension de l'implication et de l'explication, tantôt dans le quotidien du faire, pliée dans des questions financières, techniques, administratives, concrètes ; tantôt dépliée dans le calme de l'observation questionnante, de l'élaboration cognitive, de l'écriture réflexive.

14 C'est dans cette posture d'entre-deux que j'occupais une place prépondérante dans la mise en œuvre de l'expérimentation de recherche-action au sein de la plateforme d'EEDD. Pendant trois ou quatre réunions, nous avons tenté de répondre à nos questions initiales en lançant quelques pistes d'actions à mener : organiser une journée 
d'échange pour démystifier la recherche auprès des praticiens de l'éducation à l'environnement, faire un état des lieux des chercheurs bretons et de leurs objets de recherche, lancer une recherche action collective ... Tout cela demandait néanmoins des financements que beaucoup des institutions estimaient ne pas avoir pour ce type d'action. Pourtant, en 2011, la DREAL inscrivit la recherche dans son appel à projets, proposant aux associations qui le souhaitaient de constituer un dossier de demande de subvention sur une question de recherche-action. Ces recherches seraient accompagnées par un chercheur. Or, il était tard dans l'année, et malgré les courriers envoyés aux différentes universités de Bretagne, il ne nous était plus possible de trouver ce chercheur susceptible d'être intéressé par une "recherche de plein air " (Souchard, 2013, p.3). La mission me fut confiée, sous la forme d'une subvention attribuée à Echos d'Images, mon association employeuse. Entre-temps, j'étais devenue maitresse de conférences associée, contractuelle à mi-temps, à l'IUT de Tours (Université François-Rabelais), discipline des sciences de l'éducation. J'ai donc 'accompagné les quatre associations retenues dans le cadre de l'appel à projets de la DREAL, comme je le faisais avec les étudiants en apprentissage à l'écriture d'un mémoire universitaire.

15 Je connaissais bien les quatre associations. Notre grande proximité culturelle facilita notre coopération. Nous partagions nos engagements dans les mêmes réseaux. Nous possédons les mêmes savoirs associatifs, «combinaison de savoirs d'usage, de savoirs techniques et de savoirs militants» (Nez, 2009, p. 3). Notre différence principale résidait dans mon origine réflexive issue des sciences de l'éducation, alors qu'euxmêmes étaient plutôt formés aux sciences de l'environnement. «L'hybridation » de nos savoirs (Souchard, 2013) pris moins de temps à opérer que si réellement nos mondes avaient été distanciés. Peu d'asymétrie entre nous si ce n'est nos socles de savoirs scientifiques et les intentions de ces recherches, ce qui constituait l'écart suffisant pour faire émerger le dialogue, le questionnement et le tiers productif.

C'est sans doute aussi ce qui explique que, parmi la diversité des formes d'accompagnement relevées par Penven $(2013$, p. 3) allant de l'engagement militant à visée émancipatrice à la prestation de conseils, en passant par la conduite de recherches partenariales, je me suis située de façon intuitive dans la première, adoptant une posture d'accompagnatrice formatrice, cherchant à ce que les associations s'approprient les méthodes de la recherche. Ajoutant à cela mon habitude pédagogique et le manque d'appartenance à un laboratoire de recherche, la posture de la maïeutique éclairée de théories fut ma méthode d'accompagnement. Nous n'avions qu'une année pour travailler. Je leur ai donc apporté des articles scientifiques utiles, et les ai aidés à produire et rationaliser leurs propres questionnements, outils d'investigation, analyse des résultats, m'installant dans cette fonction du tiers qui facilite la mise en mots de l'expérience et interroge les certitudes. Ceci se réalisa au fil de cinq réunions que j'allais animer dans chacune des associations avec l'équipe ou la personne impliquées dans la recherche-action, et de nombreux échanges téléphoniques ou électroniques.

\section{Description de l'expérimentation}

17 Les quatre associations retenues par la DREAL (parmi peu de propositions) occupent une place importante sur la Bretagne. Elles possèdent une grande quantité d'adhérents, 
engagent de nombreuses actions en faveur de l'environnement, du développement durable ou de l'éducation populaire, actions sur lesquelles elles communiquent en interne et en externe au travers leurs propres revues, leurs sites web ou des articles de presse. Elles se positionnent régulièrement dans le débat public, et possèdent une expertise reconnue dans leur domaine d'intervention. Le montage du dossier d'intention fut une évidence pour elles. Elles avaient déjà pour projet de mener l'expérimentation innovante retenue pour la recherche-action. Certains avaient conduit des actions préparatoires à celle-ci. VivArmor Nature et le réseau Cohérence avaient même été en partenariat d'étude avec des scientifiques (Institut Universitaire Européen de la Mer pour VivArmor Nature, et l'écopsychologue Anne Versaille pour Cohérence). Le financement DREAL et l'accompagnement d'un chercheur arrivèrent comme une aubaine pour eux, ce qui facilita grandement l'entrée en démarche de recherche.

Les quatre expérimentations étaient centrées sur un même type de questionnement, celui de la mise en œuvre innovante et de l'efficacité transformatrice d'une démarche pédagogique. Cette question émergeait bien d'un problème de société repéré par les acteurs :

- Cohérence, réseau associatif pour le développement durable (et ses partenaires sur le projet: Nature et culture, CPIE Morlaix Trégor) se demande s'il est possible d'initier des agendas 21 dans les foyers.

- VivArmor nature, association d'étude de la nature et de protection de l'environnement dans les Côtes-d'Armor constate, alors que la pêche à pied est une activité pratiquée par près de 2 millions de personnes en France, que 8 pêcheurs sur 10 ne connaissent pas la réglementation faute d'information et d'encadrement, nuisant ainsi à la préservation de la faune et de la flore.

- Les Petits Débrouillards de Bretagne, association de médiation scientifique et technique, relèvent le manque de participation des habitants dans les projets de rénovation urbaine alors qu'ils sont les premiers concernés.

- Bretagne vivante, association d'étude et de protection de la nature en Bretagne, observe la difficulté qu'ont les mondes de l'agriculture et de l'environnement à dialoguer dans un contexte de restauration et de conservation de la biodiversité.

Le tableau 1 présente une synthèse des recherches-actions entreprises. 
Tableau 1 : Synthèse des recherches-actions

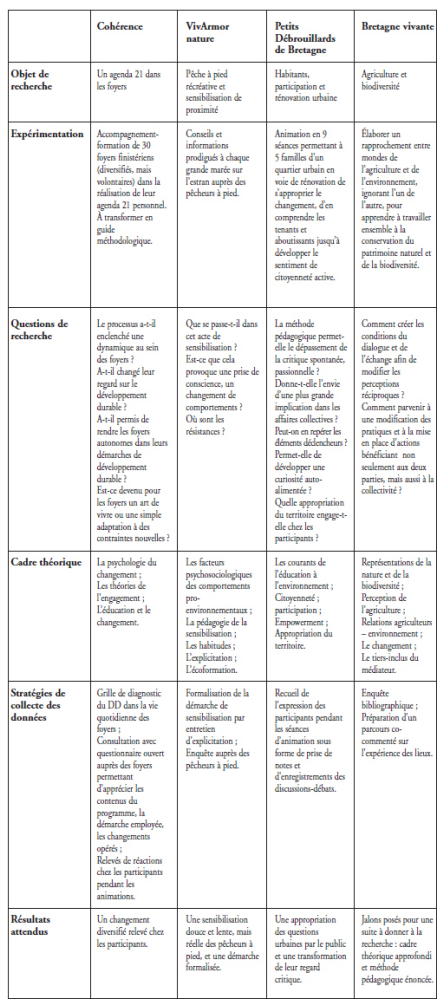

Les niveaux d'engagement dans la recherche par les associations furent importants en termes d'intérêt, d'implication subjective, de propositions. Ils se distinguent néanmoins les uns des autres sur le plan de la relation que chaque association entretient avec la recherche tout le long du processus :

- VivArmor Nature souhaita d'emblée un partenariat dans lequel chacun conserve son expertise et sa fonction : pour l'association, son rôle d'animation sur l'estran et d'expertise maritime et pour le chercheur, son rôle de mise en forme de la recherche. L'association estimait obtenir des résultats plus objectifs, donc fiables et plus facilement valorisables si les animateurs ne s'impliquaient pas eux-mêmes dans la collecte et l'analyse des données. Je produisais donc et faisais valider auprès d'eux le cadre théorique, les formes de collecte d'information, une partie de leur traitement et de la rédaction du rapport de rechercheaction.

- A l'inverse, l'animateur des Petits Débrouillards de Bretagne s'investit totalement dans la démarche de recherche. Romain Sabardin produisit lui-même tous les éléments nécessaires à l'étude et à l'analyse de l'expérimentation mise en place. Il s'appropria les articles et ouvrages qui cadraient le questionnement. Il rédigea lui-même un beau «mémoire de recherche» dans lequel les références d'auteurs éclairent ses postures d'animateur et les résultats de recherche.

- Entre les deux, et par «manque de temps» diront-ils, l'équipe de Cohérence me laissa apporter, digérer, présenter les éléments théoriques, tout en s'en servant lors des réunions comme balises à la production collective de la méthodologie de recherche et de l'analyse des résultats. L'un d'eux, Julian Pondaven, avait une expérience de recherche et nourrissait les échanges de ses propres savoirs scientifiques.

- Luc Guihard de Bretagne Vivante avait la sensation de partir de loin et de manquer de temps. Pour autant, il adopta d'emblée le même désir d'appropriation de la démarche, souhaitant lire par lui-même, me sollicitant pour des échanges à partir de ses lectures. Nous 
nous installions dans la lenteur d'investigation et de discussion conceptuelle. Une rencontre avec Dominique Bachelart (maitresse de conférence en sciences de l'éducation) cristallisa la recherche sur le "parcours co-commenté", comme outil d'enquête sur l'expérience des lieux et comme technique de rencontre-formation entre deux protagonistes pratiquant les mêmes milieux, mais sous deux visions et approches différentes. Son ouvrage «Berger transhumant en formation» (Bachelart, 2002) lui ouvrit l'horizon des approches multiréférentielles, cliniques et ethnographiques. L'année se termina sans que l'expérimentation puisse être mise en place, mais avec le projet de poursuivre dans un partenariat avec des chercheurs en sciences humaines de l'Université de Brest.

21 Pendant ce temps, la plateforme continuait de se réunir, de travailler, d'établir des passerelles avec d'autres instances intéressées par les mêmes questions de recherche partenariale, notamment le service de l'appel à projets ASOSC du Conseil régional, la plateforme de recherches-actions Périscope ${ }^{2}$ et Océanopolis de Brest souvent impliqué dans des recherches. Ensemble, nous avons élaboré une journée de valorisation des recherches-actions dont les intentions étaient de communiquer sur les résultats obtenus, de rassembler praticiens et chercheurs, et d'impulser des envies de partenariats de recherche. La journée s'est articulée autour de tables rondes invitant les associations à témoigner de leurs recherches-actions, deux interventions de chercheurs offrant discussion et généralisation à partir des présentations, et, au milieu de la journée, un "speed-meeting» pendant lequel chercheurs, praticiens et institutions se sont rencontrés, se sont présentés, rapidement, comme embarqués dans une joyeuse danse du balais. Une troupe de théâtre de médiation scientifique s'est immiscée entre les présentations, se saisissant de propos d'intervenants, les détournant, les caricaturant sur un mode clownesque provoquant éclats de rire, légèreté et dédramatisation de la scène.

Cette forme de restitution de recherches, peu académique, n'obtint pas les résultats escomptés. Le taux de participation fut faible. Peu de chercheurs répondirent à l'invitation. Les résultats présentés par les acteurs reçurent de vives critiques par quelques-uns des chercheurs présents, estimant le travail non recevable en termes de recherche. Les associations, en effet, le présentèrent avec leur culture de communication habituelle, rendant compte de l'expérience pédagogique, mais pas de l'expérience de recherche. Ces mises en partage ressemblèrent d'abord à des mises en abîme. Sans doute étions-nous dans ce que Christine Audoux et Anne Gillet nomment "l'épreuve de la traduction", "la traduction vue comme une série d'interactions permettant la mise en réseau d'acteurs, de connaissances, de productions, etc. (Callon, 1986, 2001 ; Latour, 1988, 1989), [...] comme une interprétation de langage débouchant sur un sens partagé (Ricoeur, 2004)» (2011, p. 3-4). Il était certainement fort ambitieux de notre part d'imaginer possible un tricotage immédiat des différentes cultures en présence. Identités, rôles, représentations, référentiels de savoirs, langage étaient sans doute trop éloignés pour qu'un dialogue puisse émerger. Nous savons combien toute construction d'un système nouveau passe par des ruptures, des bouleversements, qui ne peuvent atteindre une forme que si les interactions accèdent à quelques stabilisations (Morin, 1977). VivArmor, Cohérence et Les Petits Débrouillards ont dit avoir noté un contact nouveau dans leur carnet d'adresse, mais sans que cela n'ait donné de suite. Il eut sans doute fallu continuer de tisser des fils entre les deux sphères, accompagner peut-être les associations dans leur propre prospection de chercheurs. 


\section{Formation à la recherche-action ou réelles recherches- actions?} produit de la connaissance. La réponse de Franck Delisle (Vivarmor) fut, sans hésitation, « oui », car selon lui, la réussite de la diffusion et de la démultiplication de la démarche et de ses résultats l'atteste. Julian Pondaven (Cohérence) et Romain Sabardin (Petits Débrouillards) sont plus prudents et, sans les nommer, distinguent le savoir personnel (et professionnel) du savoir académique.

Le fait d'avoir explicité la démarche, de l'avoir écrite, m'a permis d'apprendre, de m'améliorer. Je continue de réfléchir quand je suis sur l'estran auprès des pêcheurs (...). Je suis passé de l'intuitif à la mise en mots. J'ai compris des choses avec les concepts, les facteurs qui influencent la sensibilisation par exemple. (FD) Pour moi, ça a été très positif en termes de prise de recul sur ma pratique, sur ma posture d'animateur que j'avais de façon intuitive. Le fait de travailler avec des apports théoriques sur la participation citoyenne, l'éducation à l'environnement, ça a donné du sens. (...) ça a été un vrai enrichissement personnel et professionnel. (...) On maîtrise mieux le milieu dans lequel on intervient. (RS)

On a pris conscience de certaines choses et ça a impacté l'orientation du projet. (...) dans le secteur de l'environnement militant, on découvre les outils de la psychologie sociale pour agir. On connaît l'éducation, mais pas les leviers 
psychologiques. On est d'habitude dans la culpabilisation. Avec la recherche-action on a modifié nos pratiques. (JP)

29 À l'instar du modèle élaboré par Kolb en 1984, il semble que les acteurs se soient trouvés embarqués dans cette boucle d'apprentissage qui, partant de l'expérience concrète, transforme les savoirs et savoir-faire par une observation réfléchie puis une conceptualisation abstraite réintroduite ensuite dans la pratique. Michel Fabre (1994, p. 145), en explorant la notion d'expérience chez Hegel, décrit ce travail qui « consiste en un mouvement dialectique par lequel la conscience pose un objet, l'examine et l'évalue (en évaluant cette évaluation elle-même) : actes qui produisent un savoir de l'objet, lequel savoir devient un nouvel objet de savoir... »..

Si le savoir individuel est indéniable dans le processus, qu'en est-il du savoir scientifique?

Je ne suis pas sûr d'avoir participé à la production de connaissances scientifiques. J'ai partagé les résultats de la recherche-action avec mes collègues, au sein de l'association. Je l'ai présentée aussi lors d'une assemblée générale de l'association nationale des Petits Débrouillards. (RS)

Est-ce qu'on a été innovant? Je ne suis pas sûr. J'imagine que des gens comme toi ou Anne Versaille, vous saviez déjà cela. Par contre, pas les militants environnementaux. Et c'est pour cela que j'essaie de transférer aux collègues. (...) La recherche-action a permis de valider notre méthode, de théoriser, mais nous devons aujourd'hui vulgariser. (JP)

31 Entre le savoir personnel et le savoir scientifique, s'immisce le savoir individuel généralisable et socialisable, en capacité d'être transmis. Éducateurs, formateurs, médiateurs, ce savoir les concerne d'emblée.

J'ai beaucoup repris d'éléments issus de la recherche-action dans le second projet. Je sais embarquer le public et l'amener de l'intérêt individuel à l'intérêt collectif maintenant. L'action a permis d'agir comme un levier pour la collectivité. (RS)

Le plus difficile à évaluer est le passage des savoirs socialisés dans sa propre arène de légitimité à celle de l'arène scientifique, une arène "dans laquelle les partenaires peuvent faire valoir les résultats et leur implication » (Audoux et Gillet, 2011, p. 8). Audelà de la journée de rencontre avec des chercheurs, nous n'avons rien fait pour élaborer cette arène élargie qui aurait favorisé la validation scientifique des recherches: pas d'articles à paraître dans des revues scientifiques (celui-ci est le premier), pas d'édition d'ouvrage. L'idée a été évoquée en réunion de plateforme, mais par manque de temps, de financement, d'implication plus engagée des partenaires, de difficulté par le passage à l'écrit d'un rapport co-rédigé entre acteurs et chercheur, le travail s'est arrêté avant cette étape décisive de communication en direction de la communauté scientifique.

33 En lien avec ces explications, cela aurait aussi supposé, comme le dit Pascal Galvani, « une revalorisation des savoirs expérientiels et d'action par rapport aux hiérarchies sociales dominantes » (1999, p. 30). Les acteurs eux-mêmes, imprégnés de la norme de scientificité, doutent de cette reconnaissance. L'épreuve ne suffit pas à faire la preuve. La recherche n'échappe pas à « l'idéologie de l'évaluation, comme nouveau dispositif de servitude volontaire » estiment Gori et Del Volgo (2009, p. 11). Et ces psychosociologues de poursuivre : "la production scientifique se mesure par la publication des résultats scientifiques. Les méthodes d'évaluation des publications utilisent habituellement les critères d'évaluation des revues scientifiques, c'est-à-dire leur «Audimat ", leur « popularité » dans une communauté de recherche » $(2009$, p. 18). Nous en sommes loin 
avec ces productions qui pour la plupart sont devenues des outils méthodologiques sans être passés par la rédaction d'un compte rendu de recherche. Romain Sabardin a rédigé un beau mémoire, resté dans les comptes rendus d'activité de l'association.

C'est sans doute la difficulté que j'ai eue d'accompagner ce travail jusqu'à ce terme scientifique. Prosaïquement, pour des raisons personnelles, j'ai quitté la Bretagne, démissionné du Conseil d'Administration du REEB, et par voie de conséquence, interrompu mon investissement dans la plateforme bretonne d'EEDD. Le contrat qui engageait les associations (dont la mienne) avec la DREAL n'engageait pas d'action de communication. Cela devait être un travail de la plateforme ... resté sans suite.

\section{Conclusions}

De par les visées mêmes de l'éducation à l'environnement, souvent ancrées dans un discours socialement critique, la recherche semblerait trouver sa niche dans des démarches partenariales, compréhensives, constructivistes et interactionnistes. Romain Sabardin (Petits Débrouillards) l'exprime ainsi : " avoir un lien étroit avec un laboratoire de recherche devrait faire partie de notre quotidien. Seul, je n'y serais pas allé, ou je ne serais pas allé si loin. Il faudrait que ça s'inscrive comme un passage obligé, que la prise de recul fasse partie intégrante de notre boulot. » Avec cette expérimentation nous ne sommes pas si loin de la définition que donne Nadine Souchard (2013, p. 3) de la recherche partenariale :

Partant d'un phénomène social qu'ils [les acteurs] construiraient en problème social, ils tenteraient de proposer des réponses en conduisant des expérimentations sociales. Pour conduire ce travail collectif, ils n'inscriraient pas leur démarche en référence à des disciplines et à des normes académiques, mais solliciteraient pour des appuis méthodologiques et théoriques des chercheurs professionnels qui saisiraient l'opportunité de conduire une recherche de "plein air» qui leur permettrait non seulement de répondre à une demande sociétale, mais aussi de recueillir des données de qualité sur des questions sociales émergentes et d'engager des formes d'intervention sociologique originales.

Plusieurs difficultés peuvent être pointées :

- Le manque de confiance personnelle en ma crédibilité vis-à-vis d'une communauté savante aux règles strictes en matière de recherche ;

- Une proximité culturelle du chercheur avec les associations qui, si elle a facilité le dialogue et l'entrée dans le travail, a pu ralentir le processus de recherche, flouter les contraintes académiques, empêcher d'aller jusqu'à la reconnaissance des pairs chercheurs ;

- Une relation timide des acteurs associatifs à la recherche, un manque de confiance dans leurs propres capacités à acquérir une légitimité sur la connaissance produite en dehors de sa communauté d'appartenance habituelle, sauf à laisser faire le travail par le chercheur ;

- La concordance des temps entre la recherche et l'action, l'une qui s'installe sur plusieurs années, l'autre qui doit faire vite, par projets annuels ;

- Le manque de financement pour sortir des injonctions sociales de production par l'action. Chaque journée d'intervention est comptabilisée dans le budget de l'association, qui n'a aucun argent supplémentaire à consacrer à la recherche dans un monde professionnel aujourd'hui en grande difficulté de survie.

37 Pour autant l'expérience n'est pas négative. Si la connaissance construite est restée au sein du monde associatif, elle n'en est pas moins réelle. "Le problème de la reconnaissance et de la formulation des différents types de savoirs n'est pas nouveau 
dans les démarches de recherche-action » nous dit Pascal Galvani $(1999$, p. 26) : «Sans renier les critères spécifiques des savoirs académiques de recherche, la rechercheaction doit intégrer et reconnaître d'autres types d'expression du savoir (Barbier, 1996, p. 74-75). Pour que s'instaure un croisement des savoirs, il faut que chaque acteur les reconnaisse en lui-même et dans les autres (Héber-Suffrin, 1992)». Ce dont est convaincu Franck Delisle (Vivarmor), « le clivage scientifique / association peut sauter. On peut être chercheur dans le cadre associatif », mais il est le seul à avoir confié le travail du chercheur au chercheur.

\section{BIBLIOGRAPHIE}

Aspe, C., Jacqué, M. (2012). Environnement et société. Paris : Éditions de la Maison des sciences de l'homme

Audoux, C., Gillet, A. (2011). Recherche partenariale et co-construction de savoirs entre chercheurs et acteurs : l'épreuve de la traduction. Revue Interventions économiques 43, Pratiques, analyses et enjeux de la recherche partenariale. Récupéré de https:// interventionseconomiques.revues.org/1347

Bachelart, D. (2002). Berger transhumant en formation : pour une tradition d'avenir. Paris : L'Harmattan, Collection « Défi-formation ».

Barbier, R. (1996). La recherche-action. Paris : Economica, Collection « Anthropos ».

Bussières, D. et Fontan, J-M. (2011). La recherche partenariale : point de vue de praticiens au Québec, SociologieS, Dossiers « Les partenariats de recherche ». Récupéré le 30 octobre 2013 de http://sociologies.revues.org/3670

Collectif breton d'éducation à l'environnement (2015). Plan régional d'action pour développer l'Éducation à l'environnement en Bretagne. Récupéré de : www.eau-loire-bretagne.fr/ espace_educatif/sensibiliser_et_eduquer/conventions_regionales/Plan-ere-bretagne.pdf Cottereau, D. (2001). Formation entre terre et mer. Alternance écoformatrice, Paris : Éditions L'Harmattan, Collection «Écologie et formation »

Cottereau, D., Le Goff, Y. (2012). Étude de faisabilité de la mise en œuvre d'un état des lieux national de l'éducation à l'environnement et au développement durable. Ministère de l'Écologie, du Développement durable, des Transports et du Logement, Commissariat général au développement durable, Délégation développement durable, France.

Fabre, M. (1994). Penser la formation, Paris : Presses universitaires de France

Galvani, P. (1999). Fertilisation croisée des savoirs et ingénierie d'alternance socio-formative. Le programme de recherche-formation-action Quart Monde/Université. Revue Française de pédagogie, $\mathrm{n}^{\circ} 128$, p. 25-34.

Goffin, L. (1998-1999). Pour une recherche en éducation relative à l'environnement centrée sur l'objet partagé. Éducation relative à l'environnement : Regards - Recherches - Réflexions, vol. 1, p. 41-61. 
Gori, R., et Del Volgo M-J. (2009). L'idéologie de l'évaluation : un nouveau dispositif de servitude volontaire? Nouvelle revue de psychosociologie, «La passion évaluative », sous la direction de Gilles Amado et Eugène Enriquez, $n^{\circ} 8$ p. 11-26.

Latour, B. (2004). Politiques de la nature, Comment faire entrer les sciences en démocratie? Paris : La Découverte.

Morin, E. (1977). La Méthode, Tome 1, La nature de la nature. Paris : Éditions du Seuil.

Nez, H. (2009). « La mobilisation des savoirs citoyens dans les dispositifs d'urbanisme participatif ", Communication aux Premières journées doctorales sur la participation du public et la démocratie participative, ENS-LSH, Lyon, 27-28 novembre.

Penven, A. (2013). La fabrique du social, croisement des savoirs et apprentissages coopératifs. Socio-logos. Revue de l'association française de sociologie. Récupéré le 15 novembre 2013 de http:// socio-logos.revues.org/2756.

Sauvé, L. (1998-1999). Pour une ERE réflexive. Éducation relative à l'environnement : Regards Recherches - Réflexions, vol. 1, p. 7-10.

Schön, D. (1994). Le praticien réflexif. À la recherche du savoir caché dans l'agir professionnel. Montréal : Éditions Logiques.

Souchard, N. (dir.) (2013). La fabrique du social, expérimentation et innovation, Tome 1. Rennes : Collège coopératif en Bretagne.

Storrie, T. (1994). La citoyenneté, un auto-apprentissage institutionnel. Dans Blanc, M., Mormont, M., Rémy, J. et Storrie, T., Vie quotidienne et démocratie. Pour une sociologie de la transaction sociale (p. 235-260). Paris : Éditions L'Harmattan.

\section{NOTES}

1. PERISCOPE : Plateforme d'Échanges et de Recherches Interdisciplinaires entre Sciences et Société Civile Organisée pour le Pilotage et l'élaboration de l'Expertise. PÉRISCOPE a pour objectif la création et la pérennisation d'une plateforme de recherche collaborative et interdisciplinaire visant à créer de l'innovation scientifique et sociale à l'interface entre sciences et société à l'échelle de la Bretagne. PÉRISCOPE est soutenu par l'Institut Universitaire Européen de la mer (IUEM) dans le cadre du Labex Mer et par la Région Bretagne dans le cadre du programme ASOSC. 2. Présentation de l'appel à projets ASOSC : «En partenariat avec les acteurs institutionnels de la recherche (universités, grands organismes, grandes écoles), la Région Bretagne est partie prenante de la promotion de la recherche et de la production d'innovations scientifiques et technologiques au bénéfice de la société régionale. Afin d'encourager pleinement l'édification d'une véritable société de la connaissance, la Région estime également nécessaire de jouer un rôle actif dans l'appropriation des sciences par la société civile et le développement des relations entre le monde scientifique et les citoyens-nes. Déjà, de nombreux acteurs de la société civile (associations, syndicats, groupes de citoyens), souvent éparpillés et disposant de peu de moyens, développent une expertise propre dans des domaines scientifiques touchant leur vie quotidienne, constituant peu à peu un "tiers-secteur " scientifique complémentaire de la recherche institutionnelle et de la recherche privée. À travers le présent appel à projets, la Région Bretagne souhaite soutenir l'émergence de ce tiers-secteur et favoriser la mise en place de programmes de recherche-action ayant pour but de traiter (et si possible de résoudre) des problématiques élaborées en réponse à des besoins et des enjeux sociétaux (problématiques de nature sociale, culturelle, économique ou environnementale). » 


\section{RÉSUMÉS}

Cet article présente un dispositif de recherches-actions associatives dans le champ de l'éducation relative à l'environnement accompagné par une chercheuse, elle-même salariée associative. Il en présente les acteurs, le processus et les résultats obtenus, soulevant les difficultés de sa mise en œuvre. Les freins à la validation et à la légitimation d'une telle initiative sont également abordés.

This article presents a non-formal sector action-research program in the field of environmentrelated education, guided by a research worker who is a voluntary sector employee herself. It presents actors and processes, while the results show the difficulties of its implementation. It also observes the disincentives to its validation and legitimization.

\section{INDEX}

Mots-clés : éducation à l'environnement, praticien réflexif, recherche action, savoirs, milieu associatif

Index géographique : France

Keywords : environmental education, reflective practitioners, action research, knowledge, associations

\section{AUTEUR}

\section{DOMINIQUE COTTEREAU}

Dominique Cottereau est à mi-temps maitresse de conférence associée à l'Université de Tours, département Carrières sociales de l'IUT. Elle y enseigne l'éducation à l'environnement. Son autre mi-temps se déroule au sein de l'entreprise coopérative Oxalis où elle occupe une fonction de consultante - formatrice en ERE, intervenant auprès des associations et collectivités territoriales sur des actions de développement de projets, d'évaluation et d'étude, de conception d'outils pédagogiques. 